\title{
Lévy flights in external force fields: from models to equations
}

\author{
D. Brockmann ${ }^{\mathrm{a}}$, I.M. Sokolov ${ }^{\mathrm{b}, *}$ \\ a Max-Planck-Institut für Strömungsforschung, Göttingen, Germany \\ ${ }^{\mathrm{b}}$ Institut für Physik, Humboldt-Universität zu Berlin, Invalidenstraße 110, D-10115 Berlin, Germany
}

Received 13 December 2001

\begin{abstract}
We consider different generalizations of the Fokker-Planck equation (FPE) devised to describe Lévy processes in potential force fields. We show that such generalizations can proceed along different lines. On one hand, Lévy statistics can emerge from the fractal temporal nature of the underlying process, i.e., a high variability in the rate of microscopic events. On the other hand, they may be a direct consequence of the scale-free spatial structure on which the process evolves. Although both forms considered lead to Boltzmann equilibrium, the relaxation patterns are quite different. As an example, generalized diffusion in a double-well potential is considered.
\end{abstract}

(c) 2002 Elsevier Science B.V. All rights reserved.

\section{Introduction}

Random walk processes leading to anomalous diffusion are adequate for describing various physical situations. The continuous time random walk (CTRW) model of Scher and Montroll [1] for instance, leading to strongly subdiffusive behavior, was a milestone in understanding photoconductivity in strongly disordered and glassy semiconductors. Due to its simplicity, this model was recently employed to investigate aging phenomena typical for glasses and related complex systems [25]. On the other hand, Lévy-flight models [6], leading to superdiffusion, are adequate for the description of phenomena ranging from transport in heterogeneous catalysis [7], self-diffusion in micelle

\footnotetext{
${ }^{*}$ Corresponding author.

E-mail address: igor.sokolov@physik.hu-berlin.de (I.M. Sokolov).
}

systems [8], reactions and transport in polymer systems under conformational motion [9], transport processes in heterogeneous rocks [10] and the behavior of dynamical systems [11] to flight paths of albatrosses [12] and even human eye-movements [13]. Closely related models appear in the description of economic time series [14]. Lévy-related statistics were observed in hydrodynamic transport [15], and in the motion of gold nanoclusters on graphite [16]. In addition, mixed models were proposed in which slow temporal evolution (described by Scher-Montroll CTRW) is combined with the possibility of Lévy-jumps, so that in general both sub- or superdiffusive behavior can arise $[17,18]$. Similarily, processes often referred to as Lévy-walks are based on long-tailed jumps in combinations with a time cost to perform them, see for example [6,19]. Lévy-walks were introduced in [20] to explain accelerated diffusion in Josephson junctions and related chaotic systems [21]. 
Typically, the corresponding processes are described on a stochastic level, such that the incorporation of boundary conditions, time or position dependent forces leads to considerable mathematical difficulties.

Concerning ordinary diffusion and transport in a stochastic system, the Fokker-Planck equation (FPE) is an adequate instrument for solving boundary value or time-dependent problems [22]. The FPE is an equation for the probability density function (pdf) $p(x, t)$ of the particle's position at time $t$ and reads

$$
\begin{aligned}
\frac{\partial p(x, t)}{\partial t} & =\mathscr{L}_{\mathrm{FP}} p \\
& =-\nabla(\mu f(x, t) p(x, t))+\kappa \Delta p(x, t) .
\end{aligned}
$$

Here $\mathscr{L}_{\mathrm{FP}}$ denotes the Fokker-Planck operator, $\mu$ and $\kappa$ are the mobility and the diffusion coefficient (assumed to be constant) and $f(x, t)$ is the external force acting on the particle. Note that since the mobility and the diffusion coefficient are connected via the Einstein relation, $\kappa=k T \mu \equiv \mu / \beta$, the equation effectively contains only one free parameter. Alternatively, Eq. (1) may be recast into a different form in the case of a potential force $f(x, t)=-\nabla \Phi(x, t)$

$$
\begin{aligned}
\frac{\partial p(x, t)}{\partial t}=\kappa[ & \mathrm{e}^{-\beta \Phi / 2}\left(\frac{\partial}{\partial x}\right)^{2} \mathrm{e}^{\beta \Phi / 2} p \\
& \left.-p \mathrm{e}^{\beta \Phi / 2}\left(\frac{\partial}{\partial x}\right)^{2} \mathrm{e}^{-\beta \Phi / 2}\right],
\end{aligned}
$$

the equivalence of which to Eq. (1) is easily checked by expanding the differentiations. Obviously, the stationary solution of the FPE (if it exists) is the Boltzmann distribution, $p(x) \propto \exp$ $(-\beta \Phi)$. Inserting this form into Eq. (2) one readily infers that the two terms entering with opposite signs are equal and cancel.

In order to investigate analogous systems incorporating anomalous diffusion, various generalizations of the FPE were proposed. Concerning subdiffusion, the appropriate generalizations can be obtained from CTRW models, for a general survey see [27], as well as from an approach based on fractional master equations [28]. Concerning superdiffusion (Lévy flights), such generalizations typically involve changing from a gradient and Laplacian to the corresponding fractional derivative. For example, a pure Lévy-flight can be described by means of the fractional diffusion equation [23-25]

$$
\frac{\partial p(x, t)}{\partial t}=\kappa_{\alpha} \Delta^{\alpha / 2} p(x, t),
$$

where $\Delta^{\alpha / 2}$ is a fractional Laplacian, a linear operator, whose action on a function $f(x)$ in Fourier space is described by $\Delta^{\alpha / 2} f(x)=-\left(k^{2}\right)^{\alpha / 2} f(k)=$ $-|k|^{\alpha} f(k)$. The coordinate representation of this operator is discussed in Appendix A. The solution of Eq. (3) in Fourier space reads

$G(k, t)=\exp \left(-\kappa|k|^{\alpha} t\right)$,

which in coordinate space corresponds to a symmetric Lévy stable distribution

$G(x, t)=\frac{1}{(\kappa t)^{1 / \alpha}} L\left(\frac{x}{(\kappa t)^{1 / \alpha}} ; \alpha, 0\right)$

(we use here the canonical notation, see [26]). On the other hand, there is no generic way to generalize the Fokker-Planck operator containing an external force term: for example, the drift term may stay unchanged, may correspond to a symmetric or asymmetric fractional derivative, or the whole Fokker-Planck operator may be raised to the power of $\alpha / 2$. All corresponding equations have their physical meaning and applications. The properties of their solutions differ considerably. Moreover, as we proceed to show, other generalizations are also possible. The situation here is to some extent similar to one in fractal geometry: What is the generalization of the Euclidean dimension to the fractal case? Depending on the system and on the property of interest it could be the fractal dimension, the spectral dimension, or even a spectrum of different dimensions. In what follows we discuss the properties of some generalization of the FPE to the superdiffusive case leading to Lévy flights in the force-free limit. We will focus on two complementary situations, namely one in which superdiffusion stems from fractal temporal properties of the underlying microscopic dynamics and another in which the spatial structure is responsible for enhanced diffusion. We mostly concentrate on the generaliza- 
tions of the forms, Eqs. (1) and (2) which lead to a Boltzmann distribution in equilibrium. We discuss the physical implications of the corresponding equations and some properties of their solutions. As a physically most interesting application, a fractional generalization of a Kramers problem in a double-well potential is considered. Let us first turn to the situations which can be considered as stemming from the fractal temporal behavior.

\section{Lévy processes as stemming from subordination}

An important property of symmetric Lévy processes is the fact that they are subordinated to ordinary Brownian motion: the corresponding pdf can be represented in the form:

$$
\begin{aligned}
p(x, t) & =\frac{1}{t^{1 / \alpha}} L\left(\frac{x}{t^{1 / \alpha}} ; \alpha, 0\right) \\
& =\int_{0}^{\infty} \frac{1}{\sqrt{2 \pi \tau}} \exp \left(-\frac{x^{2}}{2 \tau}\right) \frac{1}{t^{2 / \alpha}} L\left(\frac{\tau}{t^{2 / \alpha}} ; \frac{\alpha}{2}, 1\right) \mathrm{d} \tau,
\end{aligned}
$$

where $L(x ; \alpha / 2,1)$ is an extreme (one-sided) Lévy distribution of index $\alpha / 2$ [26].

The variable $\tau$ is called the operational time of the process. The interpretation of Eq. (6) is that Lévy flights can be considered as stemming from a highly irregular sampling trajectories generated by simple diffusion (random walk). This type of temporal behavior plays a very important role in many situations encountered in physics, such as an equilibrium phase transitions [29]. For a review see [30] and references therein.

Here, the trajectory of diffusion (a random walk in a discrete case or a Wiener path in a continuous situation) is parameterized by the operational time $\tau$ (say, the number of steps of the random walk), which itself is a random function of the (physical) time. The random process $\tau(t)$ is a process with positive increments, and the distribution of $\tau(t)$ is given by a one-sided Lévy law. Thus, the increase $\Delta \tau$ of the operational time per physical time unit is given by a one-sided Lévy-distribution, $p(\Delta \tau)=$ $L(\Delta \tau ; \alpha / 2,1)$ having a power-law tail, $p(\Delta \tau) \propto$ $\tau^{-1-\alpha / 2}$.
The corresponding situation is depicted in Fig. 1. Here a Lévy-flight of $N=100$ steps is generated as follows. One generates a sequence $\left\{n_{i}\right\}(i=1,2, \ldots, N)$ of the numbers of steps between the observation instants and plots a realization of a simple random walk of $M=\sum_{i=1}^{N} n_{i}$ steps. The turning points of a Lévy-flight are then the positions of a random walker after $m_{k}$ steps, $m_{k}=\sum_{i=1}^{k} n_{i}$. Fig. 1 corresponds to a process subordinated to a random walk under the operational time given by

$p\left(n_{i}\right)=\frac{1}{\sqrt{\pi} n_{i}^{3 / 2}} \exp \left(-\frac{1}{2 n_{i}}\right)$.

The overall pdf of the process $p(x, t)$ is then given approximately by a Cauchy distribution

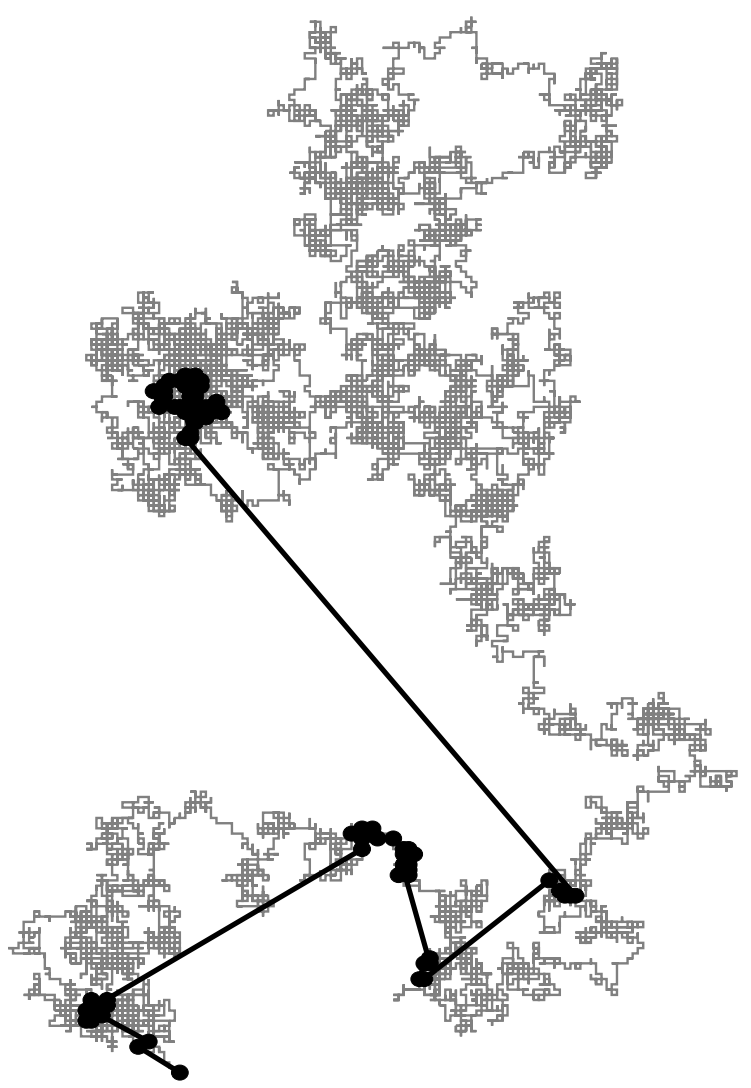

Fig. 1. The mechanism of subordination: Lévy flights can be considered as stemming from a (highly inhomogeneous) random sampling of a simple random walk. 


$$
\begin{aligned}
p(x, t)= & \sum_{n=0}^{\infty} \frac{1}{\sqrt{2 \pi n}} \exp \left(-\frac{x^{2}}{2 n}\right) \\
& \times \frac{t}{\sqrt{\pi} n^{3 / 2}} \exp \left(-\frac{t^{2}}{2 n}\right) \\
& \simeq \int_{0}^{\infty} \frac{1}{\sqrt{2 \pi n}} \exp \left(-\frac{x^{2}}{2 n}\right) \\
& \times \frac{t}{\sqrt{\pi} n^{3 / 2}} \exp \left(-\frac{t^{2}}{2 n}\right) \mathrm{d} n \\
& =\frac{t}{\pi\left(t^{2}+x^{2}\right)} .
\end{aligned}
$$

Although this continuous approximation is valid here, some care must be taken in the general case when sums over steps in random walks are replaced by integral, see for example [31]. Applying the subordination procedure to the general, biased diffusive process, one is lead to the generalization of the FPE, in which the entire FokkerPlanck operator is raised to the power $\alpha / 2$ :

$\frac{\partial p}{\partial t}=-\left(-\mathscr{L}_{\mathrm{FP}}\right)^{\alpha / 2} p$

as shown in [32]. The generalized Fokker-Planck operator $\mathscr{L}_{\alpha}=-\left(-\mathscr{L}_{\mathrm{FP}}\right)^{\alpha / 2}$ commutes with $\mathscr{L}_{\mathrm{FP}}$ and shares with it the same set of the eigenfunctions $\phi_{i}$. The corresponding eigenvalues $\Lambda_{i}$ of the operator $\mathscr{L}_{\alpha}$ are connected with those of $\mathscr{L}_{\mathrm{FP}}$ via $\Lambda_{i}=-\left(-\lambda_{i}\right)^{\alpha / 2}$. Since the eigenvalues $\lambda_{i}$ of $\mathscr{L}_{\mathrm{FP}}$ are real and nonpositive, this is true for the $\Lambda_{i}$ as well. Eq. (9) follows from the subordination procedure just in the same way as a Cauchy distribution followed from a Gaussian distribution in the example given above. Let us consider a spectral representation of the solution of the FPE

$g(x, \tau)=\sum_{i} \phi_{i}(x) \mathrm{e}^{-\left|\lambda_{i}\right| \tau}$.

The subordination procedure applied to this solution leads to

$$
\begin{aligned}
p(x, t)= & \int g(x, \tau) \frac{1}{t^{2 / \alpha}} L\left(\frac{\tau}{t^{2 / \alpha}} ; \frac{\alpha}{2}, 1\right) \mathrm{d} \tau \\
= & \sum_{i} \phi_{i}(x) \int \mathrm{e}^{-\left|\lambda_{i}\right| \tau} L\left(\frac{\tau}{t^{2 / \alpha}} ; \frac{\alpha}{2}, 1\right) \mathrm{d} \tau \\
& \times \sum_{i} \phi_{i}(x) \mathrm{e}^{-\left|\lambda_{i}\right|^{\alpha} t}
\end{aligned}
$$

due to a well-known property of the Laplacetransforms of one-sided Lévy distributions [26]. Note that the eigenfunction $\phi_{0}$ corresponding to a zero eigenvalue (if any) is a stationary distribution, which is the same (i.e., a Boltzmann distribution) for the normal and for the generalized process. The spectral decomposition of solutions of Eq. (9) given by Eq. (11) is incorporated in our numerical procedure for the solution of superdiffusive FPE in a potential field considered in Section 4.

Eq. (9) can also be interpreted within the Langevin scheme, which clarifies the circumstances under which Boltzmann statistics may or may not appear when superdiffusive generalizations of the FPEs are introduced. Let us first consider a purely diffusive situation without external force. In the continuous limit (corresponding to averaging over the time periods which are short enough to consider all parameters constant but during which many steps of a random walk are performed), the system's development in its operational time is given by a Langevin equation

$\frac{\mathrm{d}}{\mathrm{d} \tau} x(\tau)=\sqrt{2 \kappa} \xi(\tau)$,

where $\xi(\tau)$ is a $\delta$-correlated Gaussian noise with zero mean and with unit dispersion. Subordination requires that

$\frac{\mathrm{d} \tau}{\mathrm{d} t}=\lambda(t)$

where $\lambda(t)$ is a one-sided Lévy process of index $\alpha / 2$. The resulting process is thus described by a Langevin equation

$\frac{\mathrm{d} x}{\mathrm{~d} t}=\frac{\mathrm{d} \tau}{\mathrm{d} t} \frac{\mathrm{d}}{\mathrm{d} \tau} x(\tau)=\sqrt{2 \kappa} \lambda(t) \xi(\tau(t))$,

where $\tau(t)$ is a monotonously increasing random function which is changing slowly on the scale on which $\xi(\tau)$ is correlated. This suggests that the random process $\xi(\tau(t))$ is a Gaussian one, has zero mean and dispersion

$$
\begin{aligned}
\left\langle\xi(\tau(t)) \xi\left(\tau\left(t^{\prime}\right)\right)\right\rangle & =\delta\left(\tau(t)-\tau\left(t^{\prime}\right)\right) \\
& =(\mathrm{d} \tau / \mathrm{d} t)^{-1} \delta\left(t-t^{\prime}\right) \\
& =\lambda(t)^{-1} \delta\left(t-t^{\prime}\right),
\end{aligned}
$$

indicating that the process $x(t)$ can be formally represented by a Langevin equation 
$\dot{x}(t)=\sqrt{2 \kappa \lambda(t)} \eta(t)$,

where $\eta(t)$ is a $\delta$-correlated Gaussian noise with zero mean and unit dispersion. From this equation, a FPE of the type of Eq. (3) follows along the usual lines.

Physically, such strongly inhomogeneous behavior can be attributed to random fluctuations of the mobility or of the temperature or of the both: $\kappa(t)=\kappa \lambda(t)=k \mu(t) T(t)$. Let us return to the full process described by a Langevin equation

$\dot{x}(t)=\mu f+\sqrt{2 \mu k T} \xi(t)$

with a potential force $f(x, t)=-\nabla U(x, t)$. Here the situations corresponding to the fluctuations in temperature and to ones in mobility differ vastly. Keeping the temperature constant and letting $\mu$ fluctuate leads to a process subordinated to a biased random walk, which is described by a Langevin equation

$\dot{x}(t)=\mu(t) f+\sqrt{2 \mu(t) k T} \xi(t)$,

where $\mu(t)$ is now a strongly fluctuating random function, whose distribution is given by a one-sided Lévy law. The corresponding pdf is governed exactly by a fractional FPE, Eq. (9) derived above. This equation shows a relaxation to a normal Boltzmann distribution, which is not surprising since the corresponding scheme describes a system showing detailed balance at given, fixed temperature $T$. Note that although the equilibrium properties of the system are usual, its relaxation properties are not, see [33] for a detailed discussion. Thus, it does not exhibit linear response to a constant external field, and, moreover, since the corresponding pdf neither possesses the second moment nor scales, no reasonable generalization of the Einstein's relation exists. The same is valid for the model of topological superdiffusion discussed below. On the other hand, as we proceed to show, the behavior of both models in confining potentials such as a harmonic or a double-well one, is not as exotic (vide infra).

The situation under fluctuating temperature is vastly different: a system with fluctuating temperature must not show Boltzmann statistics at equilibrium (if any). Let us mention that under certain circumstances the distributions given by
Langevin equations with fluctuating parameters approach ones given by Tsallis statistics [34], i.e., ones stemming from a thermodynamic construct in which the entropy is nonextensive and thus the concept of temperature can not be introduced in a usual way.

In our case, fluctuations in temperature do not affect the first term, i.e., the deterministic motion and lead (after performing usual steps) to a fractional Fokker-Planck equation (FFPE) in the form:

$\frac{\partial p}{\partial t}=-\frac{\partial}{\partial x}(\mu f p)+\kappa \Delta^{\alpha / 2} p$,

whose solutions are discussed in [35].

\section{Topologically induced superdiffusion}

Complementary to the situation discussed in the previous section in which superdiffusion originates from fractal temporal behavior, we will discuss the fractional generalization of the FPE adequate for the description of diffusion on scale-free structures. Contrary to the situation investigated in the previous section in which superdiffusion is caused by a fractal sampling of an otherwise continuous path, we will focus on the limiting behavior of a master equation describing a Markovian jump process in which transitions are caused by thermal hopping between energetically different states on a topologically complex structure [9].

As a model system, we consider a heteropolymer in solution depicted in Fig. 2. We focus on the dynamics of a test particle performing thermally activated motion along the linear chemical coordinate axis $x$ of the polymer. If the polymer is fixed at full extend, thermal activation causes the particle to hop at a rate $1 / \tau_{\mathrm{D}}$ from a given site to a neighboring site with a probability determined by the energy difference within a given mononer pair. However, a polymer in solution is subjected to thermal conformational changes occurring on a time scale $\tau_{\mathrm{G}}$. The conformational changes allow sites which are far apart along the chemical sequence to come close in Euclidian space, as indicated in Fig. 2: for simplicity, a Gaussian chain (corresponding to a Rouse dynamics) can be con- 

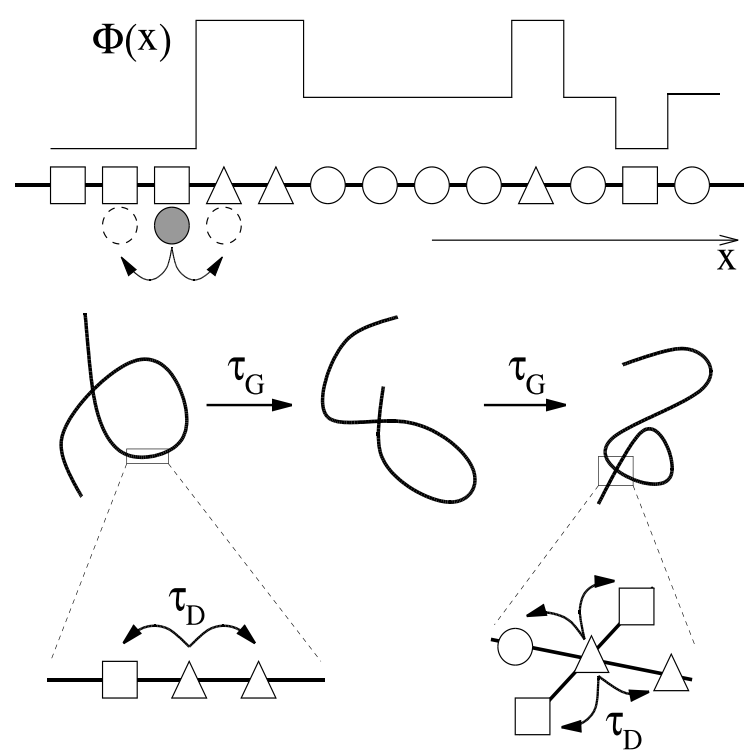

Fig. 2. Random walk of a particle on a heterogeneous polymer subjected to fast conformational changes. The polymer consist of different types of monomers denoted by the symbols along the chain (top). Each monomer is associated with an intrinsic potential $\Phi(x)$. Due to conformational changes, the walker may jump between two sites nearby in Euclidean space but far apart along the chemical sequence of the polymer.

sidered, moreover, the conformational changes will be taken fast enough on the scale $\tau_{\mathrm{D}}$. In this case the particle may jump from a given site $x$ to a site $y$ far apart with a rate proportional to the probability that the sites $x$ and $y$ have come close in the given time interval. In our case, the probability of such event is proportional to $|x-y|^{-3 / 2}$. As we proceed to show, the fractional generalization of the FPE corresponding to the situation depicted above differs from the ones considered so far.

The system introduced above can be modeled by a Markovian jump process governed by a temporally homogeneous master equation

$\frac{\partial p(x, t)}{\partial t}=\int \mathrm{d} y[w(x \mid y) p(y, t)-w(y \mid x) p(x, t)]$

in which the rates $w(x \mid y)$ are determined by the short time behavior of the conditional probability to make a transition $y \rightarrow x$

$w(x \mid y) \equiv \lim _{\Delta t \rightarrow 0} \frac{1}{\Delta t} p(x, t+\Delta t \mid y, t)$.
Let us mention here, that in Eq. (20) the rates $w(x \mid y)$ may be singular and may even defy normalizability. The Cauchy process for example is defined by $w(x \mid y)=1 / \pi(x-y)^{-2}$. If, on the other hand, $\int \mathrm{d} x w(x \mid y)=$ constant exists, the constant can be factored out and absorbed by a rescaling of the time. In this case the process is a pure jump process, consecutive jumps are seperated by time periods during which the random process is constant, in contrast to the Cauchy process whose realizations are discontinuous almost everywhere.

In our example the coordinate $x$ represents the chemical coordinate along the polymer chain. The reasoning above suggests a rate of the form

$w(x \mid y)=\frac{1}{\tau_{0}} \mathrm{e}^{-\beta[\Phi(x)-\Phi(y)] / 2} f(x-y)$.

The right-hand side of Eq. (21) consists of the thermal component $\exp (-\beta[\Phi(x)-\Phi(y)] / 2)$ accounting for the fact that transitions between energetically different states are less likely to occur if the potential difference $\Phi(x)-\Phi(y)$ is high. The second factor, $f(x-y)$ represents the probability of states with chemical coordinates $x$ and $y$ to be nearby in Euclidean space, i.e., this term accounts for the geometrical complexity of the system. Due to the translational invariance and symmetry of the system in Euclidean space this term is symmetric and depends on the distance $|x-y|$ only. Obviously, a system defined by a rate (21) fulfills detailed balance, characteristic of systems in thermal equilibrium. Note also that the stationary solution of Eq. (19) with the rates given by (21) is the Boltzmann distribution

$p_{\mathrm{s}}(x) \propto \mathrm{e}^{-\beta \Phi(x)}$.

It is instructive to consider a fully extended polymer of total length $L$. In this case only nearest neighbor hopping occurs, i.e.

$f(x)=\frac{1}{2}(\delta(x-\sigma)+\delta(x+\sigma))$.

In (23) $\sigma \ll L$ is the spacing between adjacent monomers. Setting $q_{t}(x) \equiv \mathrm{e}^{\beta \Phi(x) / 2} p(x, t)$ and $s(x) \equiv$ $\mathrm{e}^{-\beta \Phi(x) / 2}$ the master Eq. (19) reduces to: 


$$
\begin{aligned}
\frac{\partial p(x, t)}{\partial t}= & \frac{1}{2 \tau_{0}} s(x)\left\{q_{t}(x+\sigma)+q_{t}(x-\sigma)\right\} \\
& -\frac{1}{2 \tau_{0}} q_{t}(x)\{s(x+\sigma)+s(x-\sigma)\} \\
= & \frac{\sigma^{2}}{2 \tau_{0}} s(x)\left\{\frac{q_{t}(x+\sigma)+q_{t}(x-\sigma)-2 q_{t}(x)}{\sigma^{2}}\right\} \\
& -\frac{\sigma^{2}}{2 \tau_{0}} q_{t}(x)\left\{\frac{s(x+\sigma)+s(x-\sigma)-2 s(x)}{\sigma^{2}}\right\} .
\end{aligned}
$$

Now letting $\tau_{0}, \sigma \rightarrow 0$ such that $\sigma^{2} / \tau_{0} \rightarrow 2 D$ we obtain $(\Phi \equiv \Phi(x), p \equiv p(x, t))$

$$
\begin{aligned}
\frac{\partial p}{\partial t} & =\mathscr{L}_{\mathrm{G}} p, \\
& =\mathrm{e}^{-\beta \Phi / 2}\left(\frac{\partial}{\partial x}\right)^{2} \mathrm{e}^{\beta \Phi / 2} p-p \mathrm{e}^{\beta \Phi / 2}\left(\frac{\partial}{\partial x}\right)^{2} \mathrm{e}^{-\beta \Phi / 2},
\end{aligned}
$$

i.e., Eq. (2). Therfore, the dynamics of a particle on a fully extended polymer is governed by ordinary diffusion.

Let us now return to a flexible polymer performing conformational changes. In this case the geometrical factor in the rate $(21), f(x-y)$, follows a power-law [9]. Thence, we investigate the case of long-tailed transition rates. Let

$f(x)= \begin{cases}C_{\mu}|x|^{-(1+\mu)}, & |x|>\varepsilon>0, \\ 0, & \text { otherwise }\end{cases}$

with $C_{\mu}=\Gamma(1+\mu) \sin (\pi \mu / 2) / \pi$ and $0<\mu \leqslant 2$. Here $\varepsilon$ is the minimal jump length, which substitutes a fixed jump length $\sigma$ of our previous example. Note that we do not require $f(x)$ in (26) to be normalized to unity. Any prefactor independent of $\varepsilon$ occurs in both terms in the master Eq. (19) and can be absorbed as a time constant. The particular choice of $C_{\mu}$ will become clear below. Inserting (26) into (21) gives

$$
\begin{aligned}
\frac{\partial p(x, t)}{\partial t}=C_{\mu} & \left\{\mathrm{e}^{-\beta \Phi(x) / 2} \int_{|x-y|>\varepsilon} \mathrm{d} y \frac{\mathrm{e}^{\beta \Phi(y) / 2} p(y, t)}{|x-y|^{1+\mu}}\right. \\
& \left.-\mathrm{e}^{\beta \Phi(x) / 2} p(x, t) \int_{|x-y|>\varepsilon} \mathrm{d} y \frac{\mathrm{e}^{-\beta \Phi(y) / 2}}{|x-y|^{1+\mu}}\right\} .
\end{aligned}
$$

In the limit $\varepsilon \rightarrow 0$ (27) becomes (for notational ease we set $\left.s(x) \equiv \mathrm{e}^{-\beta \Phi(x) / 2}, q(x, t)=p(x, t) / s(x)\right)$

$$
\begin{aligned}
\frac{\partial p(x, t)}{\partial t}=\lim _{\varepsilon \rightarrow 0} C_{\mu}\left\{s(x) \int_{|x-y|>\varepsilon} \mathrm{d} y \frac{q(y, t)}{|x-y|^{1+\mu}}\right. & \left.-q(x, t) \int_{|x-y|>\varepsilon} \mathrm{d} y \frac{s(y)}{|x-y|^{1+\mu}}\right\} \\
= & \lim _{\varepsilon \rightarrow 0} C_{\mu}\left\{s(x) \int_{|x-y|>\varepsilon} \mathrm{d} y \frac{q(y, t)-q(x, t)}{|x-y|^{1+\mu}}\right. \\
& \left.-q(x, t) \int_{|x-y|>\varepsilon} \mathrm{d} y \frac{s(y)-s(x)}{|x-y|^{1+\mu}}\right\} \\
= & C_{\mu}\left\{s(x) \int \mathrm{d} y \frac{q(y, t)-q(x, t)}{|x-y|^{1+\mu}}\right. \\
& \left.-q(x, t) \int \mathrm{d} y \frac{s(y)-s(x)}{|x-y|^{1+\mu}}\right\} .
\end{aligned}
$$

Note, that in the limit the resulting rates $w(x \mid y)$ are not normalizable. Therefore, as mentioned earlier, the process is not a pure jump process in this limit. Implicitly, in the limit carried out above, not only the minimal step size $\varepsilon$ but also the typical waiting time $\tau_{w}(y)=\left(\int \mathrm{d} x w(x \mid y)\right)^{-1}$ at position $y$ vanishes, because of the singular nature of the rate $w(x \mid y)$ in this limit. However, the limit can be carried out since the resultant singularities cancel and Eq. (28) can be interpreted consistently.

The integrals appearing in (28) are symmetric fractional generalizations of the ordinary Laplacian (see Appendix A)

$$
\Delta^{\mu / 2} f(x)=\frac{\Gamma(1+\mu) \sin (\pi \mu / 2)}{\pi} \int \mathrm{d} y \frac{f(y)-f(x)}{|x-y|^{1+\mu}}
$$

and Eq. (28) can be recast into a more concise form:

$$
\begin{aligned}
\frac{\partial p}{\partial t} & =\mathrm{e}^{-\beta \Phi / 2} \Delta^{\mu / 2} \mathrm{e}^{\beta \Phi / 2} p-p \mathrm{e}^{\beta \Phi / 2} \Delta^{\mu / 2} \mathrm{e}^{-\beta \Phi / 2} \\
& \equiv \mathscr{L}_{\mathrm{G}, \mu} p .
\end{aligned}
$$

Again, the similarity to the underlying masterequation is obvious. Trivially, detailed balance is retained in the limit and the Boltzmann distribution is the stationary state of (30). Note that for $\mu=2$ we recover system (25), i.e. 
$\mathscr{L}_{\mathrm{G}, 2}=\mathscr{L}_{\mathrm{FP}}$.

However, in the range $0<\mu<2$ the operator $\mathscr{L}_{\mathrm{G}, \mu}$ does not portray a situation equivalent to any of the equations discussed above and does not correspond to any Langevin equation of the type

$\mathrm{d} X=F(X) \mathrm{d} t+\mathrm{d} L_{\mu}(t)$

in which the long-tailed influence is incorporated into the thermal fluctuations by a symmetric Lévy stable process $L_{\mu}(t)$. In [36] the difference between the fractional FPE corresponding to Lévy-stable additive noise and the topological generalization is investigated in detail.

\section{Comparison of systems in a double-well potential}

We proceed by showing that the two generalizations of the FPE leading to Boltzmann statistics in equilibrium (one based on the fractal time, another on the fractal space approach) differ strongly in their predictions concerning relaxation in the same kind of potential. For our comparison we
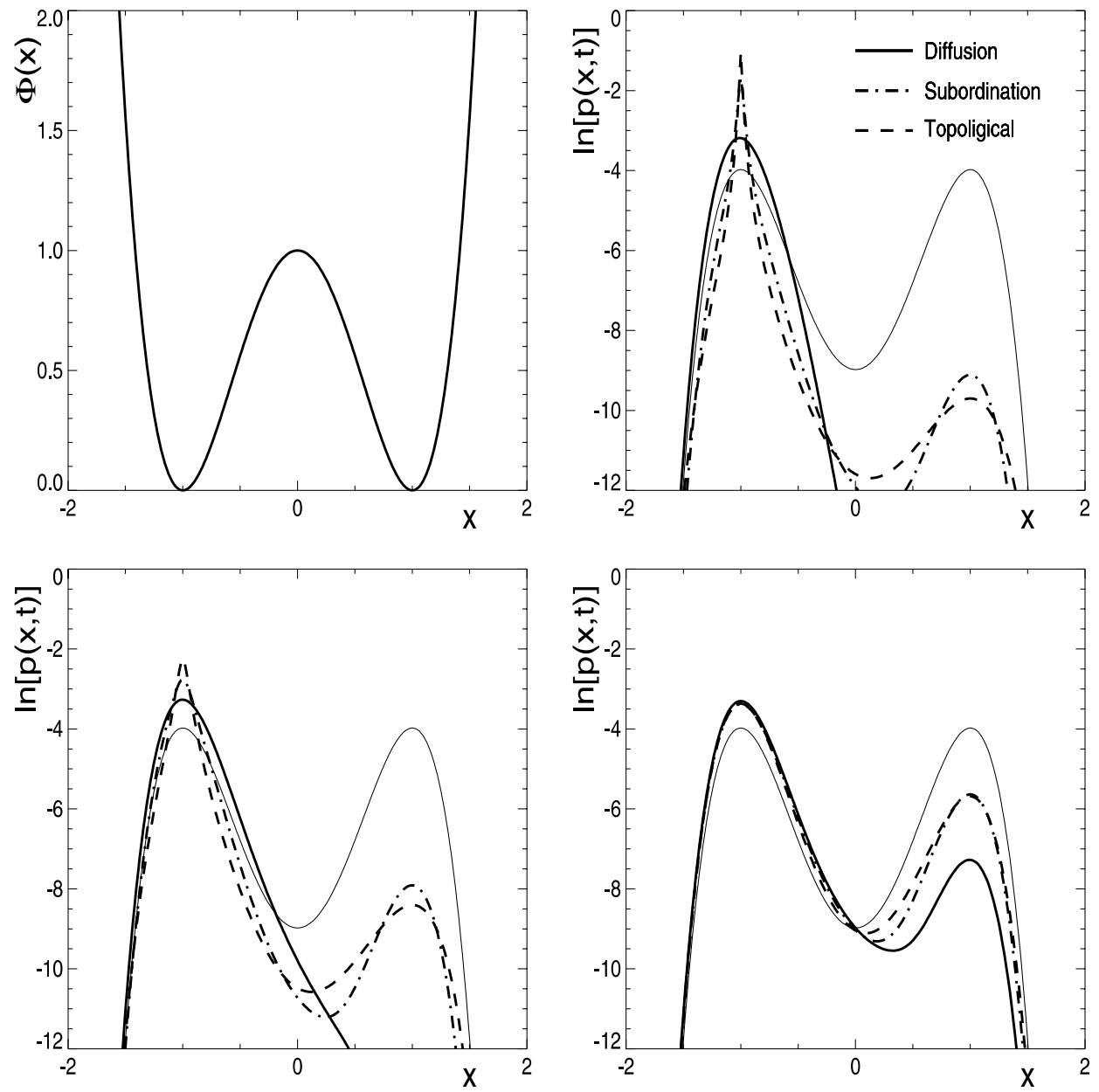

Fig. 3. Evolution of densities $p(x, t)$ in the double-well potential $\Phi(x)$ (upper left) at three different times: $t=0.01$ (upper right), 0.1 (lower left) and 1.0 (lower right) for $\beta=5.0$ and $\mu=1$ with initial condition $p(x, 0)=\delta(x+1)$ in relation to the stationary state (thin solid line). The diffusion process equilibrates quickly within the left potential well and does not pass the barrier for short times. In contrast, the superdiffusive processes transfer probability even for small times and remain peaked at $x=-1$ for much longer. 
have chosen a classical Kramers situation, i.e., diffusion in a double-well potential. As a generic double-well we chose

$\Phi(x)=x^{4}-2 x^{2}+1$

depicted in the upper left panel of Fig. 3. Initially a particle is placed at the potential minimum at $x=-1, p(x, 0)=\delta(x+1)$. The solutions $p(x, t)$ are depicted at three different times $(t=0.03,0.1,1.0)$ in Fig. 3 for a value $\beta=5.0$ and an exponent $\mu=1$. The fractional diffusion equations were numerically solved by first mapping them onto the corresponding hermitian Hamiltonian $\mathscr{H}$ and subsequent spectral decomposition $\mathscr{H}=\sum_{n} \lambda_{n} \mathscr{P}_{n}$ where the $\lambda_{n}$ and $\mathscr{P}_{n}$ are eigenvalues and projectors onto the corresponding eigenspaces. Solutions $p(x, t)$ were then expressed in term of these quantities.

Let us first note that the time scales of the relaxation processes for the case of normal diffusion and for Lévy-processes differ vastly. The typical times of the concentration equilibration (being the inverse of the largest nonzero eigenvalue) are: $\tau_{\mathrm{C}}=20.36$ for the diffusion process $(2), \tau_{\mathrm{C}}=4.51$ for the subordination case $(\mu=1)$ and $\tau_{\mathrm{C}}=3.93$ for the case of long jumps with the same value of $\mu=1$. A much greater value of $\tau_{\mathrm{C}}$ for the ordinary diffusion process is not surprising. In a short time it equilibrates within the left potential well. However,

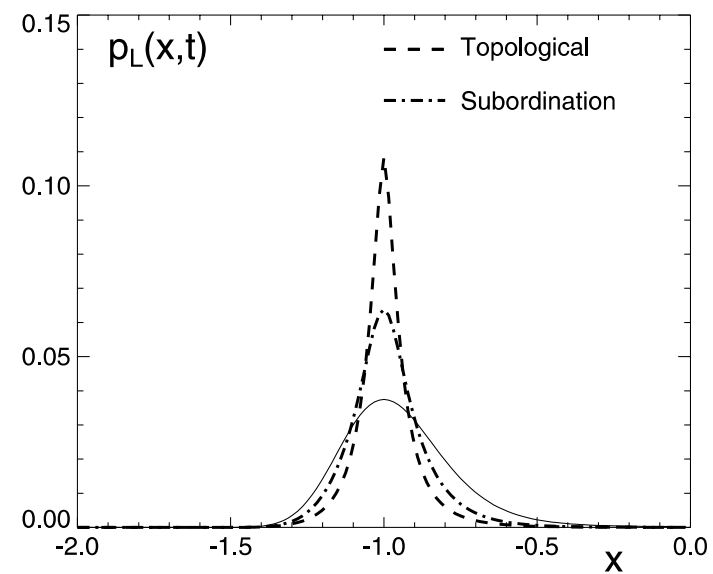

due to the continuity of its sample paths, the diffusion process accumulates probability in the right potential well only for long times. In comparison, the subordination process as well as the process governed by (30) can pass the potential maximum at $x=0$ even for short times, a direct consequence of the possibility of initiating a long jump from left to right. The possibility to transmit probability through the potential barrier is payed off by a slower equilibration within the left potential well in which $p(x, t)$ for both, subordination and topological superdiffusion stay sharply peaked around $x=-1$. This cusp-like shape in the left potential well is reminiscent of the classical Scher-Montroll picture of CTRW. Despite their qualitative similarities in the overall shape of $p(x, t)$ subordination and topological superdiffusion are quite distinct on close inspection as shown in Fig. 4.

In order to avoid huge difference in scales, we plot in Fig. 4 the reduced densities in the left and in the right potential wells, i.e., the densities which are normalized to unity on the given subinterval and for the given time:

$$
\begin{aligned}
& p_{\mathrm{L}}(x, t)=\frac{p(x, t)}{\int_{-\infty}^{0} \mathrm{~d} y p(y, t)}, \\
& p_{\mathrm{R}}(x, t)=\frac{p(x, t)}{\int_{0}^{\infty} \mathrm{d} y p(y, t)} .
\end{aligned}
$$

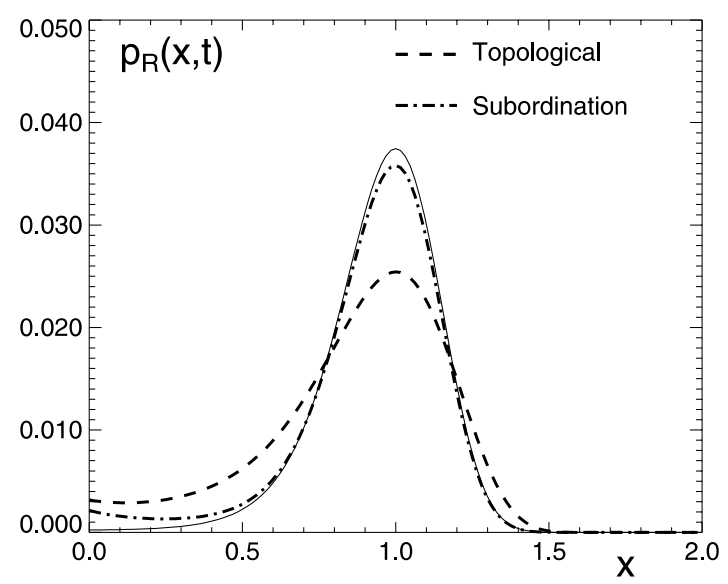

Fig. 4. The shape of $p_{\mathrm{L}}(x, t)$ (left) and $p_{\mathrm{R}}(x, t)$ (right) for subordinative and topological superdiffusion at $t=0.1, \beta=5.0$ and exponent $\mu=1$. Note the difference in scale on the left and right, respectively. Despite the fact that these processes are identical if $\Phi=0$, they respond quite differently if a potential is imposed. 
Clearly, the shapes differ strongly on both sides of the potential.

In order to reveal the differences in barrier penetration it is instructive to consider the time dependence of the total probability in the right potential well

$Q(t)=\int_{0}^{\infty} \mathrm{d} x p(x, t)$

and the rate $r(t)$ defined by

$r(t)=-\frac{1}{2} \frac{\mathrm{d} \ln Q(t)}{\mathrm{d} t}$.

Asymptotically, as $t \rightarrow \infty$ the processes investigated here evolve according to

$p(x, t) \approx p_{\mathrm{s}}(x)-\left(p_{\mathrm{s}}(x)-p_{0}(x)\right) \mathrm{e}^{\lambda_{1} t}, \quad \lambda_{1}<0$,

where $p_{\mathrm{s}}(x)$ and $p_{0}(x)$ denote the stationary and initial states of the system, respectively. Therfore, $r(t) \rightarrow-\lambda_{1}$ as $t \rightarrow \infty$, the rate approaches the

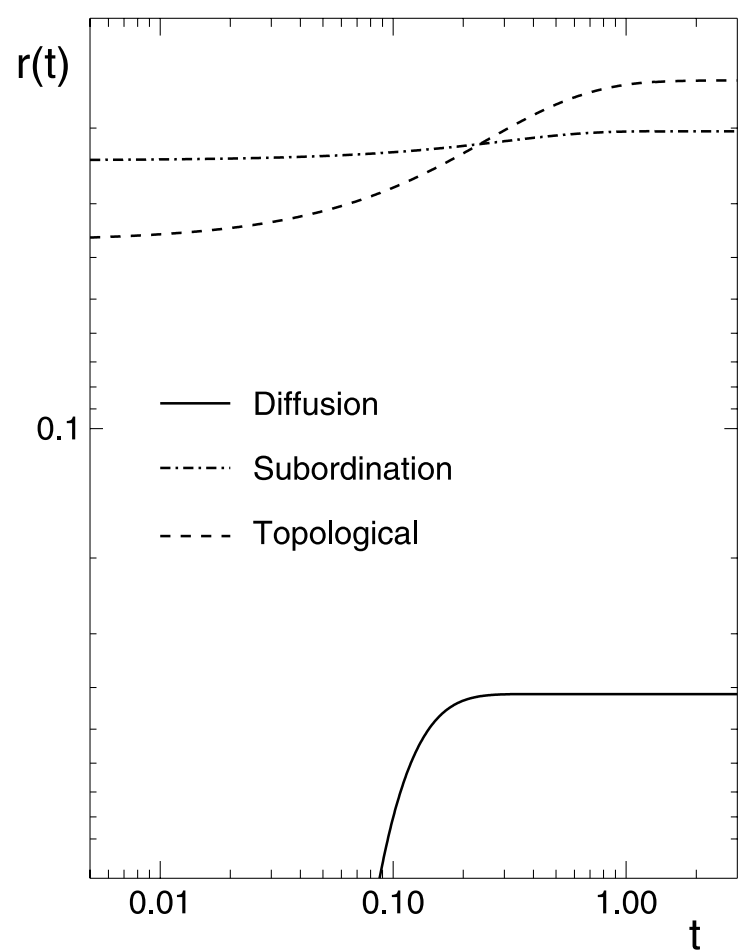

Fig. 5. The time dependent rate $r(t)$ for ordinary diffusion, subordination and topological superdiffusion in the doublewell. Parameters are $\beta=5.0, \mu=1$. inverse of the relaxation time constant of the process. In other words, the function $r(t)$ measures how fast the probability transfer across the barrier approaches the quasi-equilibrium value. For the same parameters as in Figs. 3 and 4 the rate $r(t)$ is depicted in Fig. 5. The ordinary diffusion rate is initially zero and increases as soon as the process equilibrates within the left potential well. It then levels off to a relatively small constant value proportional to the probability flux across the barrier. In contrast, for the superdiffusive systems the rate to transfer probability over the barrier is finite even for very small times. For the latter $r(t)$ increases more slowly than in ordinary diffusion. However, the asymptotic rate is higher. Note that the subordination process penetrates the barrier at relatively high but almost constant rate for the entire time, whereas for topological superdiffusion the rate is initially smaller but increases for large times beyond the subordination rate.

\section{Conclusions}

We discussed different generalizations of a FPE for the case of Lévy processes in external force fields. We show that such generalization can proceed along different lines (assuming strong temperature or mobility fluctuations or allowing for long jumps), which lead to different forms of the generalized operator. We confined ourselves to situations in which the corresponding equations are thermodynamically sound and their solutions approach Boltzmann equilibrium. The different relaxation behaviors of Lévy-processes were investigated and compared for the paradigmatic case of a double-well potential and deviations from normal diffusion were clarified.

\section{Acknowledgements}

The authors acknowledge useful discussions with J. Klafter, A. Chechkin, and W. Noyes. The financial support of the Fonds der Chemischen Industrie is gratefully acknowledged. 


\section{Appendix A. Fractional differentiation}

The operators involved in fractional differentiation are generically nonlocal. Thus, depending on the particular choice of boundary values a variety of generalizations exists, all of which suit a specific need in physical applications. Depending on the specific problem at hand different representations of fractional differential operators may be chosen. Here we concentrate on a fractional generalization, $\Delta^{\mu / 2}, 0<\mu \leqslant 2$ of the ordinary Laplace operator $\Delta$ generically encountered in superdiffusive systems. The reader is referred to [37] for additional information on fractional calculus in general.

\section{A.1. Definitions}

Incidentally, the most natural way to introduce fractional differential operators is based on the generalization of the $n$-fold iterated integral of a function $f(x)$

$$
\begin{aligned}
{ }_{a} I_{x}^{n} f(x) & \equiv \frac{1}{(n-1) !} \int_{a}^{x} \mathrm{~d} y f(y)(x-y)^{n-1} \\
& =\frac{1}{\Gamma(n)} \int_{a}^{x} \mathrm{~d} y f(y)(x-y)^{n-1}
\end{aligned}
$$

to arbitrary fractional order $\alpha>0$

$$
\begin{aligned}
& { }_{a} I_{x}^{\alpha} f(x) \equiv \frac{1}{\Gamma(\alpha)} \int_{a}^{x} \mathrm{~d} y f(y)(x-y)^{\alpha-1}, \\
& { }_{x} I_{a}^{\alpha} f(x) \equiv \frac{1}{\Gamma(\alpha)} \int_{x}^{a} \mathrm{~d} y f(y)(y-x)^{\alpha-1},
\end{aligned}
$$

where we distinguish between the right- and lefthanded fractional integrals ${ }_{a} I_{x}^{\alpha}$ and ${ }_{x} I_{a}^{\alpha}$, respectively. A fractional derivative of order $\mu$ which is frequently employed in physical applications is given by the $n$th ordinary derivative of the fractional integral of order $0<n-\mu \leqslant 1$, where $n=[\mu]+1$. In other words

$$
\begin{aligned}
& { }_{a} D_{x}^{\mu} f(x) \equiv \frac{\mathrm{d}^{n}}{\mathrm{~d} x^{n}}{ }_{a} I_{x}^{n-\mu}, \\
& { }_{x} D_{a}^{\mu} f(x) \equiv \frac{\mathrm{d}^{n}}{\mathrm{~d} x^{n}}{ }_{x} I_{a}^{n-\mu} .
\end{aligned}
$$

Note that since the distinction between left- and right-handed fractional integrations is passed on to fractional differentiation, fractional derivatives are not symmetric with respect to interchanging their subscripts. The definitions (A.4, A.5) imply that

${ }_{a} D_{x}^{m}=\frac{\mathrm{d}^{m}}{\mathrm{~d} x^{m}}=(-1)^{m}{ }_{x} D_{a}^{m}, \quad m=0,1,2, \ldots$

That is, fractional differentiation is proportional to ordinary differentiation for integer values of the exponent. ${ }^{1}$ By sequential partial integration (A.4) can be recast into

$$
\begin{aligned}
{ }_{a} D_{x}^{\mu} f(x)= & \sum_{k=0}^{n-1} \frac{f^{(k)}(a)}{\Gamma(1+k-\mu)}(x-a)^{k-\mu} \\
& +{ }_{a} I_{x}^{n-\mu} \frac{\mathrm{d}^{n}}{\mathrm{~d} x^{n}} .
\end{aligned}
$$

Therfore, the operations of fractional differentiation and integration generally do not commute. However, if we let $a \rightarrow \pm \infty$ and require that $\lim _{a \rightarrow \pm \infty} f^{(k)}(a)<\infty$ (A.4) and (A.5) yield

${ }_{-\infty} D_{x}^{\mu} f(x)=\frac{1}{\Gamma(n-\mu)} \int_{-\infty}^{x} \mathrm{~d} y \frac{f^{(n)}(y)}{(x-y)^{\mu+1-n}}$,

${ }_{x} D_{\infty}^{\mu} f(x)=\frac{1}{\Gamma(n-\mu)} \int_{x}^{\infty} \mathrm{d} y \frac{f^{(n)}(y)}{(y-x)^{\mu+1-n}}$.

In fractional diffusion equations the symmetric fractional generalization of the Laplace operator frequently appears. Its one-dimensional variant can be defined in terms of ${ }_{a} D_{x}^{\mu} f(x)$ and ${ }_{x} D_{a}^{\mu} f(x)$ by

$$
\begin{aligned}
\Delta^{\mu / 2} & \equiv-\frac{1}{2 \cos (\pi \mu / 2)}\left\{{ }_{-\infty} D_{x}^{\mu}+{ }_{x} D_{\infty}^{\mu}\right\}, \\
0 & <\mu \leqslant 2 .
\end{aligned}
$$

The particular case of $\Delta^{1 / 2}$ requires some care. Although the definition (A.9) does not give a useful result for $\mu=1$, since both numerator and denominator vanish, the limit $\mu \rightarrow 1$ can be interpreted consistently as will become clear below.

\footnotetext{
${ }^{1}$ To recover symmetry for integer values of the exponent $m$ one frequently encounters an alternative definition of the lefthanded fractional derivative, namely ${ }_{x} D_{a}^{\mu} f(x) \equiv(-1)^{n}\left(\mathrm{~d}^{n} /\right.$ $\left.\mathrm{d} x^{n}\right)_{x} I_{a}^{n-\mu}$ which includes the prefactor $(-1)^{n}$ in the definition.
} 
Partial integration of (A.7, A.8) and insertion into (A.9) yields

$$
\begin{aligned}
\Delta^{\mu / 2} f(x)= & -\frac{1}{2 \Gamma(-\mu) \cos (\pi \mu / 2)} \\
& \times \int_{-\infty}^{\infty} \mathrm{d} y \frac{[f(y)-f(x)]}{|x-y|^{1+\mu}} .
\end{aligned}
$$

Since $\lim _{\mu \rightarrow 1} \Gamma(-\mu) \cos (\pi \mu / 2)=-\pi / 2$ the operator $\Delta^{1 / 2}$ is well defined, i.e.

$\Delta^{1 / 2} f(x)=\frac{1}{\pi} \int_{-\infty}^{\infty} \mathrm{d} y \frac{[f(y)-f(x)]}{(x-y)^{2}}$.

Clearly, $\Delta^{1 / 2} \neq \nabla=\mathrm{d} / \mathrm{d} x$, the "square-root" of the Laplacian is symmetric, whereas the first derivative is not. ${ }^{2}$ The operators ${ }_{-\infty} D_{x}^{\mu},{ }_{x} D_{\infty}^{\mu}$ and $\Delta^{\mu / 2}$, possess a particularly simple form when the equation involved are Fourier transformed. If we define the Fourier transform of $f(x)$ by

$\mathscr{F}[f](k) \equiv \widetilde{f}(k)=\int \mathrm{d} x \mathrm{e}^{\mathrm{i} k x} f(x)$

the transformed Eqs. (A.7), (A.8) and (A.10) read

$\mathscr{F}\left[_{-\infty} D^{\mu} f\right](k)=(-\mathrm{i} k)^{\mu} \widetilde{f}(k)$,

$\mathscr{F}\left[D_{\infty}^{\mu} f\right](k)=(\mathrm{i} k)^{\mu} \widetilde{f}(k)$,

$\mathscr{F}\left[\Delta^{\mu / 2} f\right](k)=-|k|^{\mu} \widetilde{f}(k)$.

Thus, the Fourier transform of fractional differentiation is equivalent to a multiplication by $|k|^{\mu}$. Because of this, the investigation of equations containing operators such as $\Delta^{\mu / 2}$ is usually much easier when expressed in Fourier space. The simplicity of Eqs. (A.13), (A.14) and (A.15) is often a reason to chose the latter as definitions of fractional derivatives. On the other hand, the corresponding integral representations (A.7), (A.8) and (A.10) offer a more direct picture of the characteristic properties of the corresponding operator. For example, the Cauchy process obeys $\partial_{t} \widetilde{p}(k, t)=-|k| \widetilde{p}(k, t)$ in Fourier space, a simple equation indeed. By inverse Fourier transform this gives

\footnotetext{
${ }^{2}$ In the literature the symbol $\nabla^{\mu}$ is frequently used instead of $\Delta^{\mu / 2}$ which can lead to some confusion if $\mu=1$.
}

$$
\begin{aligned}
\partial_{t} p(x, t) & =\left(\Delta^{\frac{1}{2}} p\right)(x, t) \\
& =\int \mathrm{d} y[w(x \mid y) p(y, t)-w(y \mid x) p(x, t)], \\
w(x \mid y) & =\frac{1}{\pi(x-y)^{2}} .
\end{aligned}
$$

In contrast to the Fourier representation it is obvious from Eqs. (A.17) and (A.18) that the fractional operator $\Delta^{1 / 2}$ is nonlocal, involves singularities and appearing in the example given above describes a jump process, a fact not readily available in Fourier representation.

To complete the picture, let us mention that the form (A.15) of the fractional Laplace operator can be generalized to arbitrary dimensions $n$. Let $f(\mathbf{x})$ be a function on $\mathbb{R}^{n}$ and $\widetilde{f}(\mathbf{k})$ the corresponding Fourier transform, then $\Delta^{\mu / 2}$ defined by

$\mathscr{F}\left[\Delta^{\mu / 2} f\right](\mathbf{k}) \equiv-|\mathbf{k}|^{\mu} \widetilde{f}(\mathbf{k})$

yields upon Fourier inversion the integral representation

$$
\Delta^{\mu / 2} f(\mathbf{x})=-\frac{2^{\mu} \Gamma\left(\frac{\mu+n}{2}\right)}{\pi^{n / 2} \Gamma\left(-\frac{\mu}{2}\right)} \int_{-\infty}^{\infty} \mathrm{d}^{n} y \frac{[f(\mathbf{y})-f(\mathbf{x})]}{|\mathbf{x}-\mathbf{y}|^{\mu+n}} .
$$

\section{References}

[1] H. Scher, E.W. Montroll, Phys. Rev. B 12 (1975) 2455.

[2] C. Monthus, J.-P. Bouchaud, J. Phys. A 29 (1996) 3847.

[3] B. Rinn, P. Maass, J.-P. Bouchaud, Phys. Rev. Lett. 84 (2000) 5405.

[4] I.M. Sokolov, A. Blumen, J. Klafter, Europhys. Lett. 56 (2001) 175.

[5] I.M. Sokolov, A. Blumen, J. Klafter, Physica A 302 (2001) 268.

[6] J. Klafter, M.F. Shlesinger, G. Zumofen, Phys. Today 49 (1996) 33.

[7] O.V. Bychuk, B. O'Shaughnessy, Phys. Rev. Lett. 74 (1994) 1795; J. Chem. Phys. 101 (1994) 772.

[8] A. Ott, J.-P. Bouchaud, D. Langevin, W. Urbach, Phys. Rev. Lett. 65 (1994) 2201.

[9] I.M. Sokolov, J. Mai, A. Blumen, Phys. Rev. Lett. 79 (1997) 857;

J. Lumin. 76-77 (1998) 377. 
[10] J. Klafter, A. Blumen, G. Zumofen, M.F. Shlesinger, Physica A 168 (1990) 637.

[11] M. Bologna, P. Grigolini, J. Riccardi, Phys. Rev. E 60 (1999) 6435.

[12] G.M. Viswanathan, V. Afanasyev, S.V. Buldyrev, E.J. Murphy, P.A. Prince, H.E. Stanley, Nature 381 (1996) 413.

[13] D. Brockmann, T. Geisel (submitted).

[14] P. Sazntini, Phys. Rev. E 61 (2000) 93.

[15] T.H. Solomon, E.R. Weeks, H.L. Swinney, Phys. Rev. Lett. 71 (1993) 3975.

[16] W.D. Luedtke, U. Landman, Phys. Rev. Lett. 82 (1999) 3835.

[17] H.C. Fogedby, Phys. Rev. E 50 (1994) 1657.

[18] R. Metzler, E. Barkai, J. Klafter, Europhys. Lett. 46 (1999) 431.

[19] M.F. Shlesinger, G.M. Zaslavsky, J. Klafter, Nature 363 (1993) 31.

[20] T. Geisel, J. Nierwetberg, A. Zacherl, Phys. Rev. Lett. 54 (1985) 616.

[21] T. Geisel, A. Zacherl, G. Radons, Phys. Rev. Lett. 59 (1987) 2503.
[22] C.W. Gardiner, Handbook of Stochastic Methods, Springer, Berlin, 1997.

[23] V. Seshadri, B.J. West, PNAS 79 (1982) 4501.

[24] V. Seshadri, Phys. Rev. A 36 (1987) 892.

[25] H.C. Fogedby, Phys. Rev. Lett. 73 (1994) 2517.

[26] W. Feller, An Introduction to Probability Theory and Its Applications, vols. I and II, Willey, New York, 1971.

[27] R. Metzler, J. Klafter, Phys. Rep. 339 (2000) 1.

[28] R. Hilfer, L. Anton, Phys. Rev. E 51 (1995) 848.

[29] R. Hilfer, Phys. Rev. E 48 (1993) 2466.

[30] R. Hilfer (Ed.), Applications of Fractional Calculus in Physics, World Scientific, Singapore, 2000.

[31] E. Barkai, Phys. Rev. E 63 (2001) 046118.

[32] I.M. Sokolov, J. Klafter, A. Blumen, Phys. Rev. E 64 (2001) 021107.

[33] I.M. Sokolov, Phys. Rev. E 63 (2001) 011104.

[34] C. Beck, Phys. Rev. Lett. 87 (2001) 180601.

[35] S. Jespersen, R. Metzler, H.C. Fogedby, Phys. Rev. E 59 (1999) 2736.

[36] D. Brockmann, T. Geisel (submitted).

[37] K.B. Oldham, J. Spanier, The Fractional Calculus, Academic Press, New York, 1974. 